\title{
XP01 regulates erythroid differentiation and is a new target for the treatment of $\beta$-thalassemia
}

\author{
Flavia Guillem, ${ }^{1,2,3}$ Michaël Dussiot, ${ }^{1,2,3^{*}}$ Elia Colin,,$^{1,2,3^{*}}$ Thunwarat Suriyun, ${ }^{1,2,3}$ \\ Jean Benoit Arlet, ${ }^{1,2,3,4}$ Nicolas Goudin, ${ }^{5}$ Guillaume Marcion, ${ }^{6,7}$ \\ Renaud Seigneuric, ${ }^{6,7}$ Sebastien Causse, ${ }^{6,7}$ Patrick Gonin, ${ }^{8}$ Marc Gastou, ${ }^{3,8,9}$ \\ Marc Deloger, ${ }^{10}$ Julien Rossignol, ${ }^{1,11,12}$ Mathilde Lamarque, ${ }^{1,2,3}$ \\ Zakia Belaid Choucair, ${ }^{1,2}$ Emilie Fleur Gautier, ${ }^{3,13}$ Sarah Ducamp, ${ }^{3,13}$ \\ Julie Vandekerckhove, ${ }^{1}$ Ivan C. Moura, ${ }^{1,2,3 \dagger}$ Thiago Trovati Maciel, ${ }^{1,2,3}$ \\ Carmen Garrido, ${ }^{6,7,14}$ Xiuli An, ${ }^{15}$ Patrick Mayeux, ${ }^{3,13}$ Narla Mohandas, ${ }^{15}$ \\ Geneviève Courtois ${ }^{1,2,3 \#}$ and Olivier Hermine ${ }^{1,2,3,11 \#}$
}

Volume 105(9):2240-2249

\section{Correspondence:}

OLIVIER HERMINE

ohermine@gmail.com

Received: November 22, 2018.

Accepted: November 19, 2019.

Pre-published: November 21, 2019.

doi:10.3324/haematol.2018.210054

(C)2020 Ferrata Storti Foundation

Material published in Haematologica is covered by copyright. All rights are reserved to the Ferrata Storti Foundation. Use of published material is allowed under the following terms and conditions:

https://creativecommons.org/licenses/by-nc/4.0/legalcode. Copies of published material are allowed for personal or internal use. Sharing published material for non-commercial purposes is subject to the following conditions:

https://creativecommons.org/licenses/by-nc/4.0/legalcode, sect. 3. Reproducing and sharing published material for commercial purposes is not allowed without permission in writing from the publisher.
${ }^{1}$ INSERM UMR 1163, CNRS ERL 8254, Laboratory of Cellular and Molecular Mechanisms of Hematological Disorders and Therapeutical Implications, Paris, France; ${ }^{2}$ Imagine Institute, Université Paris Descartes, Sorbonne Paris-Cité et Assistance Publique-Hôpitaux de Paris, Hôpital Necker, Paris, France; ' ${ }^{3}$ Laboratory of Excellence GRex, Paris, France; ${ }^{4}$ Service de Médecine Interne, Faculté de Médecine Paris Descartes, Sorbonne Paris-Cité et Assistance Publique-Hôpitaux de Paris, Hôpital Européen Georges Pompidou, Paris, France; ${ }^{5}$ US24, Cell Imaging Platform, Necker Federative Structure of Research (SFR-Necker), Paris, France; ${ }^{\circ}$ INSERM, Unité Mixte de Recherche 866, Equipe Labellisée Ligue Contre le Cancer and Association pour la Recherche contre le Cancer, and Laboratoire d'Excellence Lipoprotéines et Santé (LipSTIC), Dijon, France; ' ${ }^{7}$ Faculty of Medicine and Pharmacy, University of Burgundy, Dijon, France; ${ }^{8}$ Gustave Roussy, Université Paris-Saclay, Plateforme d'Evaluation Préclinique-UMS 3655/US23, Villejuif, France; ' Université Paris 7 Denis Diderot-Sorbonne Paris Cité, Paris, France; ${ }^{10}$ Institut Curie, PSL Research University, INSERM, U 900, MINES,

ParisTech, Paris, France; ${ }^{11}$ Service d'Hématologie, Faculté de Médecine Paris Descartes, Sorbonne Paris-Cité et Assistance Publique-Hôpitaux de Paris Hôpital Necker, Paris, France; ${ }^{12}$ Département d'Hématologie, Gustave Roussy, Université Paris-Saclay, Villejuif, France; ${ }^{13}$ Institut Cochin, INSERM U1016, CNRS UMR8104, Université Paris Descartes, and Plateforme de Proteomique Paris 5 (3P5), Paris, France; ${ }^{14}$ Centre Anticancéreux George François Leclerc, Dijon, France and ${ }^{15}$ Red Cell Physiology Laboratory, New York Blood Center, New York, NY, USA

${ }^{*} \mathrm{MD}$ and EC contributed equally to this work; " $\mathrm{GC}$ and $\mathrm{OH}$ contributed equally to this work as co-senior authors. ${ }^{+}$This article is dedicated to the memory of Ivan C. Moura who passed away during its preparation.

\section{ABSTRACT}

-thalassemia major ( $\beta$-TM) is an inherited hemoglobinopathy caused by a quantitative defect in the synthesis of $\beta$-globin chains of hemoglobin, leading to the accumulation of free $\alpha$-globin chains that aggregate and cause ineffective erythropoiesis. We have previously demonstrated that terminal erythroid maturation requires a transient activation of caspase- 3 and that the chaperone Heat Shock Protein 70 (HSP70) accumulates in the nucleus to protect GATA-1 transcription factor from caspase-3 cleavage. This nuclear accumulation of HSP70 is inhibited in human $\beta$-TM erythroblasts due to HSP70 sequestration in the cytoplasm by free $\alpha$-globin chains, resulting in maturation arrest and apoptosis. Likewise, terminal maturation can be restored by transduction of a nuclear-targeted HSP70 mutant. Here we demonstrate that in normal erythroid progenitors, HSP70 localization is regulated by the exportin-1 (XPO1), and that treatment of $\beta$-thalassemic erythroblasts with an XPO1 inhibitor increased the amount of nuclear HSP70, rescued GATA-1 expression and improved terminal differentiation, thus representing a new therapeutic option to ameliorate ineffective erythropoiesis of $\beta$-TM. 


\section{Introduction}

Erythropoiesis is a multistep process leading to red cell production from hematopoietic stem cells. We have demonstrated that entry into the terminal maturation stage of erythroblasts, marked by important morphological modifications, requires a transient activation of caspase- $3 .{ }^{1}$ However, in this process, some caspase- 3 targets including GATA1, the transcription factor involved in the expression of erythroid genes like erythropoietin (EPO) receptor, Glycophorin A or globin chains, remains uncleaved. The chaperone Heat Shock Protein 70 (HSP70), which is constitutively expressed during erythroid differentiation, accumulates in the nucleus and protects GATA-1 transcription factor from caspase-3 cleavage, allowing continued terminal maturation. ${ }^{2}$ We have demonstrated that this nuclear accumulation of HSP70 did not occur in human $\beta$-TM erythroblasts, resulting in GATA1 cleavage and thus erythroblast maturation arrest and apoptosis. Indeed, in $\beta-T M$, HSP70 is sequestrated in the cytoplasm by the excess of free $\alpha$-globin chains preventing its nuclear localization. ${ }^{3}$ Likewise, terminal maturation in human $\beta$-TM could be restored by transduction of a nuclear-targeted HSP70 mutant. Thus, any regulation enabling an increase in HSP70 nuclear concentration would be an interesting therapeutic strategy to correct ineffective erythropoiesis of $\beta$-TM. However, mechanisms involved in HSP70 trafficking during human erythroid differentiation are still unknown. Thus, we focused our work on elucidating those mechanisms. We have previously shown by both confocal microscopy and immunoblot analyses that, in erythroblasts derived from $\mathrm{CD} 36^{+}$cells, EPO starvation induced a dramatic decrease in both nuclear localization of HSP70 and as a consequence GATA-1 expression. Addition of the nuclear export inhibitor Leptomycin B (LMB) ${ }^{4,5}$ prevented this phenomenon. ${ }^{2}$ These previous observations strongly suggested that HSP70 localization could be regulated mainly by its export. Exportins are proteins of the $\beta$-karyopherin group that mediate export from the nucleus to the cytoplasm of cellular proteins (cargos) or mRNAs, using the RanGTP/GDP gradient. There are seven known exportins expressed in human cells (XPO1 to XPO7) and all show preferential cargo specificity. ${ }^{6}$ It has been recently reported that the erythroid-specific isoform of exportin-7 (XPO7) was the most abundant exportin expressed at the mRNA level in very late erythropoiesis, and was involved in chromatin condensation and enucleation in murine erythroid differentiation. ${ }^{7}$ However, since XPO7 is expressed and plays a role at the later stage of erythroid differentiation, it might not be considered as a good candidate to be an exporter of HSP70.

In order to decipher which exportin(s) was/were involved in HSP70 nuclear export in erythroid progenitor, we have analyzed the expression profile of the seven different human exportins from transcriptomic and proteomic data from erythroid cells at different differentiation stages of maturation, and showed functionally that XPO1 is involved in HSP70 export and could be considered as a good target to ameliorate ineffective erythropoiesis of $\beta$-TM.

\section{Methods}

\section{Erythroid liquid culture and KPT-251 treatment}

Erythroid cells were generated in vitro from peripheral blood circulating $\mathrm{CD}_{3} 4^{+}$cells from adult patients with $\beta 0$-thalassemia major $(\beta-\mathrm{TM})$, which were collected before routine transfusion or from cord blood from healthy donors. This study was performed according to the Declaration of Helsinki with the approval from the ethics committee of our institution [Comite de Protection des Personnes (CPP) "Ile de France II"]. All patients gave written informed consent. In the first step of culture ("cell expansion"), isolated CD34 ${ }^{+}$progenitors (Miltenyi CD34 Progenitor Cell Isolation Kit) were grown in the presence of $100 \mathrm{ng} / \mathrm{mL}$ IL-6, $10 \mathrm{ng} / \mathrm{mL}$ IL3 , and $100 \mathrm{ng} / \mathrm{mL}$ SCF for 7 days. At day 7 , erythroid progenitors were switched to the second phase of culture, which allows the differentiation and maturation of erythroblasts: cells were cultured in the presence of $10 \mathrm{ng} / \mathrm{mL}$ IL-3, $50 \mathrm{ng} / \mathrm{mL} \mathrm{SCF}$, and $2 \mathrm{U} / \mathrm{mL}$ EPO in IMDM (Gibco cell culture) supplemented with 15\% BIT 9500 (Stem Cell Technologies), as previously described. Solid KPT-251 was reconstituted in DMSO. Cells were treated with KPT-251 at 100 or $1000 \mathrm{nM}$, or with DMSO alone as control condition.

\section{Cell differentiation}

Erythroid differentiation was assessed by various methods. First, morphological analysis after May-Grunwald-Giemsa (MGG) staining was used. Cells were examined under Zeiss microscope axioplan 2, Camera Oimaging OICAM FAST 1394. The number of immature (proerythroblasts + basophilic), polychromatophilic, and mature (orthochromatic erythroblasts + reticulocytes) erythroblasts was assessed in each experiment by counting approximately 200 cells in consecutive fields and expressed as a percentage of total cells. Additionally, differentiation was assessed by calculating a terminal maturation index (TMI) on MGG, defined by the number of orthochromatic erythroblasts + reticulocytes ${ }^{*} 100 /$ number of polychromatophilic cells per slide. This allowed us to better characterize the maturation arrest at the polychromatophilic stage, which is known to be a hallmark of $\beta$-TM ineffective erythropoiesis, ${ }^{8}$ and its modulation.

Flow cytometry analysis was also performed at several different times during erythroid culture after double labeling with Band3 and 4-integrin, two optimal surface markers to differentiate highly mature erythroblasts. ${ }^{9}$ PE-conjugated anti-Band3 (PE-BRIC6 conjugate, Bristol Institute for Transfusion Sciences, UK) and APC-conjugated anti- 4-integrin (Miltenyi Biotec) antibodies were used for flow cytometry (FACS Gallios). This double labeling allowed us to assess terminal erythroblastic differentiation and purify cell populations by cell sorting (BD FACS Aria II SORP). High Band3 (high band3, low $\alpha 4$-integrin), and low Band3 (low band3, high 4-integrin) gates were defined, and cells from each gate were sorted and analyzed morphologically after MGG staining.

\section{Statistical analysis}

Statistical analyses were performed with GraphPadPrism (Version 4.0 GraphPad software). Data are expressed as the mean \pm standard deviation or standard error of the mean. Paired $t$-test, or Anova Dunn's, Dunnett's, and Tukey's multiple comparison tests were used as appropriate. $P<0.05$ was considered statistically significant; lower $P$-values are indicated in the figure legends. Further information is available in the Online Supplementary Appendix.

\section{Results}

\section{XP01 can interact with HSP70 nuclear export sequence}

In our previous work, ${ }^{2}$ we demonstrated that HSP70 export mediated by EPO deprivation could be repressed by treatment with the nuclear export inhibitor LMB. 


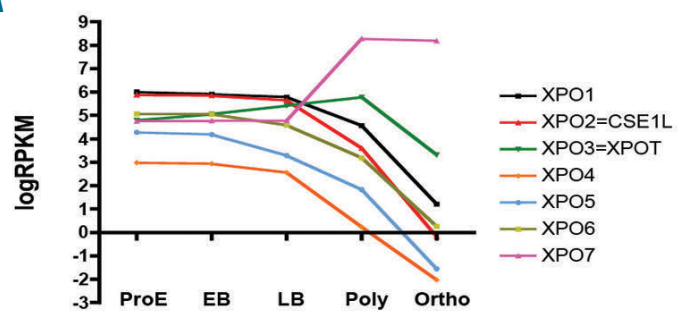

C

NES human HSP70 protein WT 394 LDVAPLSLGL ${ }^{40}$ NES human HSP70 protein mut ${ }^{394}$ LDVAPLALGL ${ }^{403}$

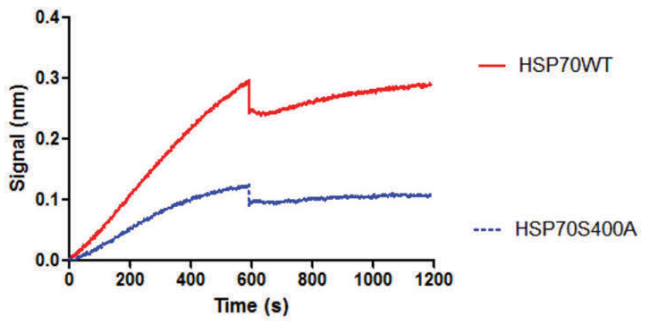

D

B

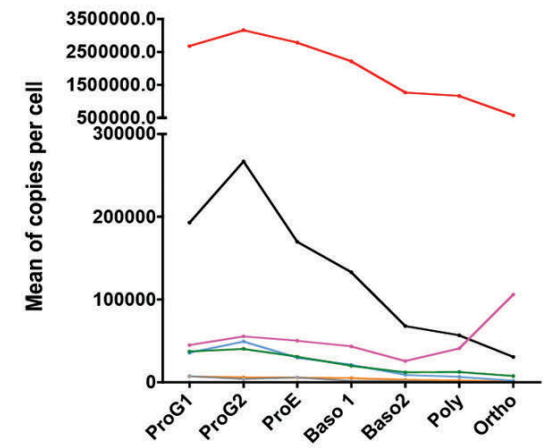

Duolink : HSP70-XPO1 complexes

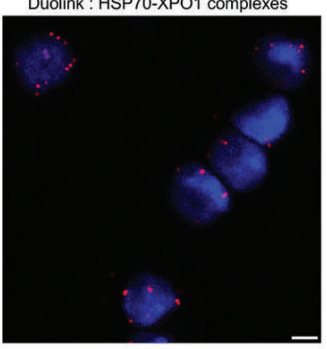

negative control (GATA1-XPO1)

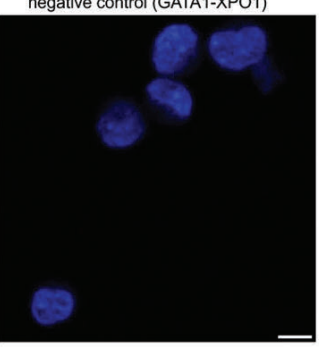

$\mathrm{F}$
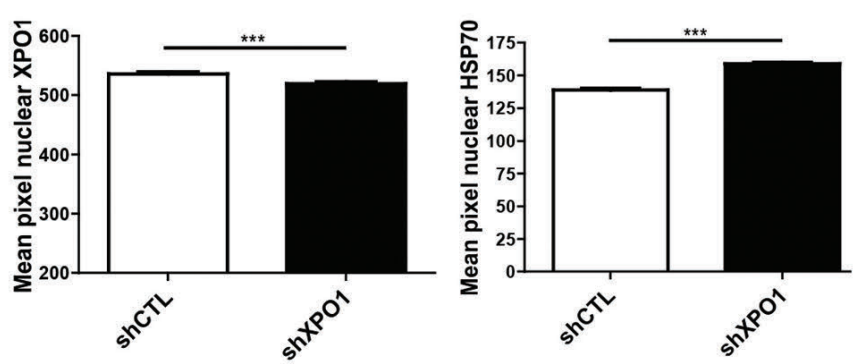

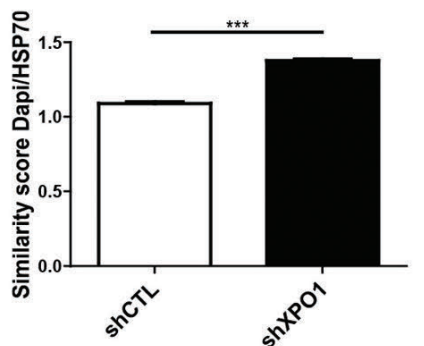

Figure 1 (previous page). In human erythroid progenitors, HSP70 is exported from the nucleus by an XP01-dependent mechanism. Expression profiles for the seven different exportins (XPO1-XPO7) during terminal erythroid differentiation in human: mRNA expression from proerythroblast stage (ProE) to orthochromatic stage (Ortho). Values are extracted from public data and presented as log of reads per kilobase of transcript per million reads (logRPKM); EB: early basophile; LB: late basophile; Poly: polychromatophilic, (A) and protein expression from progenitor stage (ProG) to orthochromatic stage (Ortho). Values are presented as mean of protein copies per cell. ProG1: BFU-E; ProG2: CFUE; Baso1: early basophile; Baso2: late basophile; Poly: polychromatophilic (B). Data are representative of three independent experiments. (C) Putative XP01 specific leucin-rich NES in the protein sequence of human HSP70 (NP_005336) at position L394-L403. The interactions between purified XPO1 and WT HSP70 as well as XPO1 with the nucleartargeted HSP70 mutant (S400A) were analyzed using BLI. WT HSP70 exhibits a much higher signal (i.e. affinity) for the ligand XPO1 compared to the nuclear HSP70 protein bearing a mutation in the NES residue S400A. Data are representative of two independent experiments. (D) Proximity of HSP70 and XP01 proteins was analyzed in CD36 ${ }^{+}$ erythroid progenitors derived from cord blood, by Duolink assay, using anti-XPO1 and anti-HSP70 antibodies (or anti-GATA1 for negative control). Red spots indicate <40 nm proximity between cellular-bound antibodies. Nuclei are stained with DAPI (blue). Images have been observed by confocal microscopy (x63 oil objective, scale bar $=5 \mu \mathrm{m})$. Data are representative of three independent experiments. (E) HSP70 and XPO1 direct interaction was demonstrated by CoIP experiments. HSP70 and XPO1 immunoblot detection is shown in total lysate (TL), in eluate from HSP70 IP and from IgG Control (IgG CTL) IP. The data are representative of three independent experiments in human erythroïd cells. (F) Erythroid progenitors from $\beta$-thalassemia major ( $\beta$-TM) patient at day 2 of CD36 culture were transduced with a shRNA specific for XPO1 or a sh scramble (shCTL). Both constructions express GFP. GFP ${ }^{+}$cells were sorted and stained with anti-HSP70 or anti-XPO1 antibodies, and DAPI. XPO1 and HSP70 nuclear expression (mean pixel) were analyzed at day 2 following transduction, by ImageStream. In addition, HSP70 nuclear translocation was evaluated by measuring the similarity score between HSP70 and DAPI nuclear stainings. Data are presented as mean \pm standard error of mean (SEM). On average, 30,000 events were collected in all experiments. $P$-values are determined by paired $t$-test. $* * * P<0.0001$. Three illustrative images (ImageStream) of ShCTL and shXPO1 conditions are presented. Cells were probed for HSP70 expression and run on the ImageStream. Bright field (white), DAPI (purple), HSP70 (green), and HSP70/DAPI composite (scale bar=7 $\mu$ m). Data are representative of six independent experiments, $\mathrm{n}=2$ different $\beta$-TM patients with $\mathrm{n}=2$ different shRNA XPO1. 
Here, we validate these previous findings using confocal microscopy and ImageStream analysis for a more quantitative approach. In normal culture conditions of cord blood-derived $\mathrm{CD}^{+} 6^{+}$erythroid progenitors at day 3, a 2hour treatment with $\mathrm{LMB}$ at $20 \mathrm{nM}$ induced an increase in HSP70 nuclear accumulation (Online Supplementary Figure $S 1 A$ and $B$ ) resulting in an increase in HSP70 nuclear/cytoplasmic ratio.

In order to determine which specific exportin was involved in HSP70 export, we first analyzed expression of the seven exportins during erythropoiesis. All of them were expressed at the mRNA and protein levels during human erythroid differentiation as shown by transcriptomic and proteomic analysis ${ }^{10,11}$ (Figure 1A and B).

Based on the proteomic database (Figure 1B), we found that exportin-2 (XPO2) is the most expressed exportin at the protein level in human erythroid progenitors. However, since primary function of XPO2 is to mediate re-export of importin- $\alpha$ from the nucleus to the cytoplasm, it is unlikely to be the candidate exportin involved in HSP70 nuclear export. In contrast, XPO1, in addition to exporting RNAs, mediates export of a broad range of cargo proteins bearing a leucine-rich Nuclear Export Sequence (NES), ${ }^{12,13}$ that include a large variety of tumor suppressor proteins (e.g. p53, p21, FOXO) and thereby fulfills all the criteria to be a good candidate in exporting HSP70. We performed in silico analysis of published data for GATA-1 chromatin immuno-precipitation (ChIP) in

Table 1. GATA-1 chromatin immunoprecipitation (ChIP) peak scores in XP01 gene and three known erythroid genes.

\begin{tabular}{llc} 
Gene name & Genomic peak location & Peak score \\
XP01 & Intragenic intron & 53 \\
& Intragenic intron & 695.35 \\
& Intragenic intron & 76.79 \\
& Intragenic intron & 94.13 \\
& Intragenic intron & 231.83 \\
& Immediate down. Intron & 62.86 \\
& Promoter & 292.11 \\
& Enhancer & 110.24 \\
& Intergenic & 66.87 \\
& Intergenic & 288.01 \\
EPOR & Intergenic & 73.56 \\
& Intergenic & 89.17 \\
& Intragenic exon & 101.5 \\
& Immediate down. Intron & 3100 \\
& Enhancer & 72.94 \\
& Enhancer & 186.49 \\
\hline HBA1 & Enhancer & 55.94 \\
& Promoter & 898.4 \\
& Promoter & 105.72 \\
& Enhancer & 1259.62 \\
& Enhancer & 313.4 \\
& Promoter & 55.51 \\
\hline And & &
\end{tabular}

Annotated peaks extracted from GATA-1 ChIP public data on human erythroid progenitors $^{14}$ analyzed with Galaxy software. Peak scores and locations for XPO1 and the known erythroid genes EPOR (erythropoietin receptor),HBA1 (Hemoglobin $\alpha-1$ ) and HBA2 (Hemoglobin $\alpha-2$ ). Peak score in MACS output corresponds to $-10 * \log 10$ pvalue of peak region. $P$-value corresponds to the probability that the candidate peak (reads enrichment) is random human erythroid cells, ${ }^{14}$ and found twelve annotated peaks for GATA-1 in the promoter of the XPO1 gene suggesting that it might be an erythroid regulated gene (Table 1). In addition, XPO1 was the second most expressed exportin in erythroblasts, and concomitantly with HSP70 nuclear accumulation, its expression was down-regulated both at the mRNA and protein levels along terminal differentiation (black line in Figure 1A and B). This hypothesis was further supported by in silico analysis showing that human HSP70 protein sequence contains a putative leucine-rich NES at position L394L400 that could interact with XPO1 (Figure 1C, NES human HSP70 protein WT).

In order to validate this hypothesis, we first showed that XPO1 interacted with HSP70 in vitro by protein-protein interaction experiments using BioLayer interferometry (BLI) (Figure 1C, red line). Furthermore, we observed a decreased binding affinity between XPO1 and HSP70 with the mutant Serine 400 Alanine (HSP70S400A) in the putative leucine-rich NES (Figure 1C, blue line). This mutant HSP70 shows restricted nuclear localization when transduced in erythroid cells. ${ }^{3}$ Thus, these results validate the importance of putative NES in the interaction of HSP70 with XPO1, and in the cellular localization of HSP70. Next, using proximity ligation assay (PLA/Duolink assay) (Figure 1D) and performing CoIP experiments in normal conditions (Figure 1E), we obtained additional evidence that this interaction is direct and occurs in vivo in $\mathrm{CD}_{3} 6^{+}$derived erythroid progenitors. Interestingly, the fraction of XPO1 protein immunoprecipitating with HSP70 upon EPO deprivation is increased as shown by CoIP experiments (data not shown).

\section{XP01 is the exporter of HSP70 in human erythroid progenitors}

To document the functional role of XPO1 in human erythropoiesis, and its putative target to treat $\beta$-TM, we first repressed XPO1 expression in human $\beta$-TM erythroblasts. For this purpose, $\mathrm{CD} 36^{+}$cells from a $\beta$-TM patient were transduced with lentiviruses expressing a XPO1 specific shRNA (shXPO1) or a sh scramble used as control (shCTL). Two days after transduction, ImageStream quantification showed that the cells transduced with shXPO1 efficiently repressed the expression of XPO1 [mean pixel nuclear

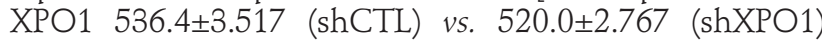
$(P<0.0001]$ (Figure 1F and Online Supplementary Figure 2A). Similar results were obtained in erythroblasts from cord blood and peripheral blood stem cells (data not shown). In agreement with our hypothesis, XPO1 repression was associated with a significant increase in HSP70 nuclear translocation [similarity score $1.090 \pm 0.012$ for shCTL vs. $1.378 \pm 0.011$ for shXPO1 $(P<0.0001)]$ and in HSP70 nuclear accumulation [mean pixel nuclear HSP70 139.1 \pm 1.176 for shCTL vs. $159.2 \pm 1.173$ for shXPO1 $(P<0.0001)$, corresponding to a $14.5 \%$ increase] (Figure $1 \mathrm{~F}$ ). Similar results were obtained using two different shRNA specifically targeting XPO1. As a control of efficacy, P53 that is a well-known target of XPO1, was also significantly increased in the nucleus (Online Supplementary Figure $2 B$ and C). Taken together, these data suggest that in human erythroid cells, HSP70 interacts with XPO1 through a leucine-rich NES region, which is required for its nuclear export and may thus be a good target to improve ineffective erythropoiesis of $\beta$-TM. 
A

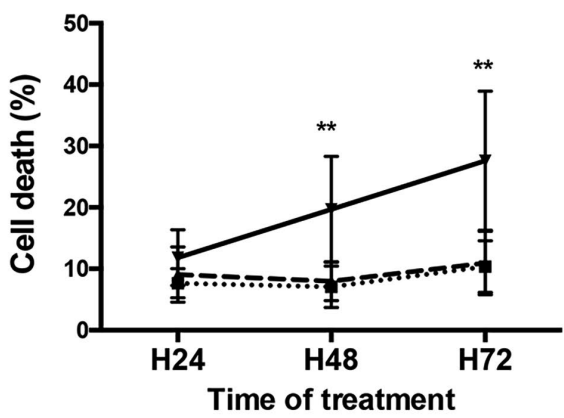

C

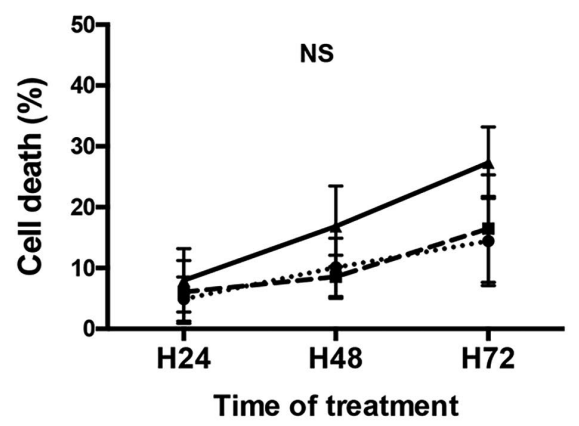

B

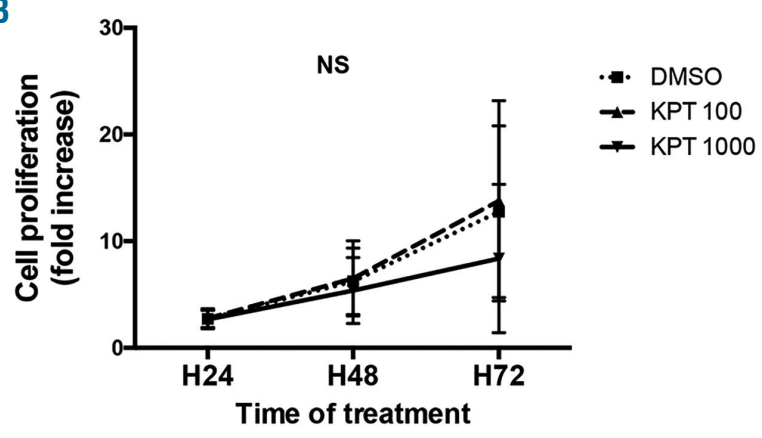

D

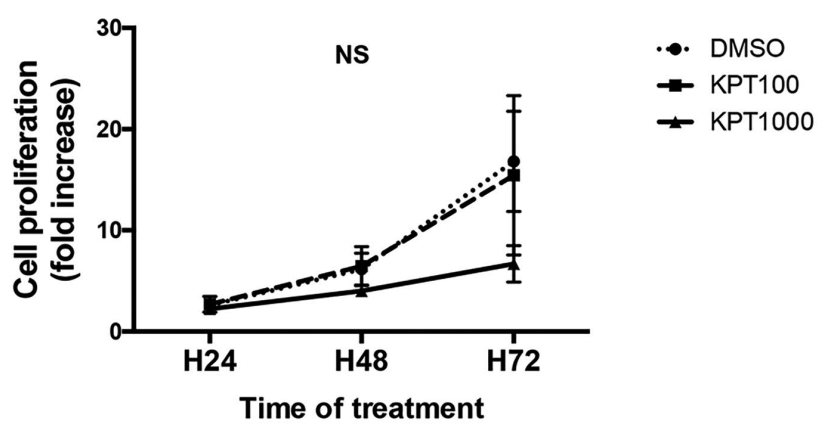

Figure 2. KPT-251 treatment has low effect on cell proliferation and cell death. Cell death and proliferation curves analysis of $\beta$-thalassemia major ( $\beta$-TM) (A and B) and cord blood (C and D) erythroid progenitors, assessed by blue trypan staining at 24, 48 and 72 hours (H) of treatment with KPT100nM, KPT1000nM, or DMSO (control). Daily mean percentage \pm standard deviation (SD) of dead cells ( $n=5$ independent experiments for $\beta$-TM and $n=3$ independent experiments for cord blood). Daily mean \pm standard deviation of cell proliferation $(n=6$ independent experiments for $\beta$-TM and $n=3$ independent experiments for cord blood). $P$-values are determined by ANOVA Dunnett's multiple comparison test $* * P<0.01$, NS: not significant.

Chemical inhibition of XP01 using KPT-251 treatment increases the amount of nuclear HSP70 and GATA-1 in $\beta$-TM erythroid progenitors

We repressed XPO1 activity using KPT-251 (Merck Millipore), a Selective Inhibitor of Nuclear Export (SINE) that specifically inhibits the formation of XPO1-cargo complex by interacting with the NES binding-groove of XPO1. KPT-251 treatment induces minimal toxicity in non-cancerous hematopoietic cells both in vitro and in vivo, ${ }^{15-19}$ with almost no effect on cell survival (Figure $2 \mathrm{~A}$ and $\mathrm{C}$ ) and a decrease in cell proliferation but not significant (Figure $2 \mathrm{~B}$ and $\mathrm{D}$ ). This is in contrast to $\mathrm{LMB}$ treatment, an inhibitor of nuclear export which is highly toxic for erythroid progenitors (data not shown). We exposed erythroid progenitors from $\beta$-TM patients, at day 4 of $\mathrm{CD}^{+} 6^{+}$ day of culture, to KPT-251 at $100 \mathrm{nM}, 1000 \mathrm{nM}$, or to DMSO (control) for 72 hours (h). As evidenced by immunoblot analyses at day 7 of $\mathrm{CD} 6^{+}$culture, the treatment resulted in a significant dose-dependent decrease in the amount of XPO1 protein compared to control (DMSO) in cytoplasmic extracts (CE) (Figure 3A). XPO1 protein decrease following KPT treatment was also observed in erythroid progenitors derived from cord blood, and significant XPO1 protein decrease can be observed in erythroid progenitors from $24 \mathrm{~h}$ of treatment (data not shown). This finding is consistent with previous studies using different primary cancer cells and cell lines. ${ }^{20,21}$ XPO1 repression by the treatment probably acts through a proteasome-dependent mechanism as reported earlier for KPT-185 and KPT-330, two other members of the KPT family. ${ }^{22,23}$ In $\beta$-TM erythroblasts, western blot analysis showed the decrease in XPO1 protein amount is associated with a significant dose-dependent increase in HSP70 protein amount in nuclear extracts (NE) (Figure 3A). Nuclear GATA1 amounts and cytoplasmic HSP70 amounts remain unchanged. These results were further confirmed by confocal analyses (Figure 3B); after a 72-h exposure to KPT-251, increases were observed in HSP70 nuclear concentration [mean fluorescence intensity (MFI) nuclear HSP70 48.27 \pm 4.6 (control), 58.85 \pm 5.2 (KPT 100 $\mathrm{nM})(\mathrm{NS})$ and $79.06 \pm 9.0(\mathrm{KPT} 1000 \mathrm{nM})(P<0.01)]$ and in HSP70 nuclear/cytoplasmic (N/C) ratio $[\mathrm{MFI}$ ratio $0.24 \pm 0.02$ (control), $0.35 \pm 0.02(\mathrm{KPT} 100 \mathrm{nM})(P<0.01)$ and $0.37 \pm 0.03$ (KPT 1000nM) $(\mathrm{P}<0.01)$. Consistent with an increase in nuclear location of HSP70, GATA-1 nuclear concentration was also increased as assessed by confocal analyses [MFI nuclear GATA-1 48.53 \pm 5.9 (control), $44.32 \pm 2.9$ (KPT $100 \mathrm{nM})(\mathrm{NS})$ and 74.17 \pm 2.8 (KPT $1000 \mathrm{nM}) \quad(P<0.01)]$ (Figure $3 \mathrm{~B})$. Quantification by ImageStream (Figure $3 \mathrm{C}$ and $\mathrm{D}$ ) further confirmed the increase in HSP70 nuclear accumulation by KPT-251 treatment [mean pixel nuclear HSP70 95.22 \pm 0.40 (control), 98.27 $\pm 0.36(\mathrm{KPT} 100 \mathrm{nM})(P<0.01)$ and $108.2 \pm 0.38(\mathrm{KPT}$ $1000 \mathrm{nM})(P<0.01)$, and of the HSP70 N/C ratio [mean pixel ratio $1.551 \pm 0.02$ (control), $1.654 \pm 0.02(\mathrm{KPT} 100 \mathrm{nM})$ $(P<0.01)$ and $1.647 \pm 0.02(\mathrm{KPT} 1000 \mathrm{nM})(P<0.01)]$ and GATA-1 nuclear expression [mean pixel nuclear GATA-1 $730.2 \pm 2.1$ (control), 756.2 $\pm 2.2(\mathrm{KPT} 100 \mathrm{nM})(P<0.01)$ and $816.5 \pm 2.1$ (KPT 1000nM) $(P<0.01)]$ induced by KPT treatment, in a dose-dependent manner. In addition, as determined by similarity score, the fraction of erythroblasts with a nuclear translocation of HSP70 was increased fol- 
A

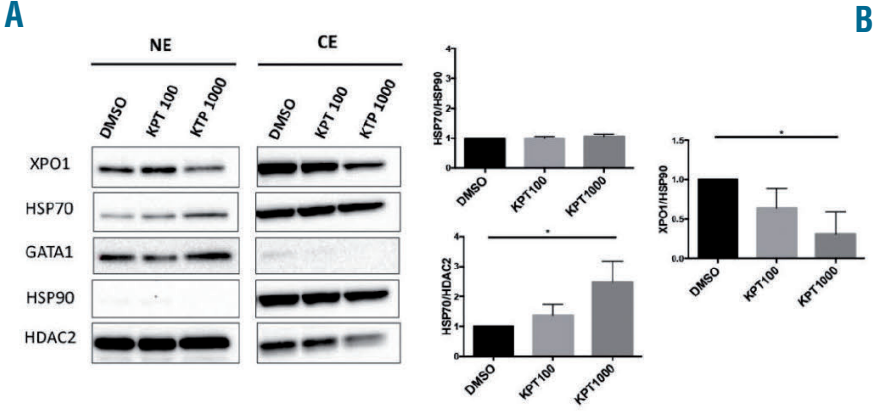

B
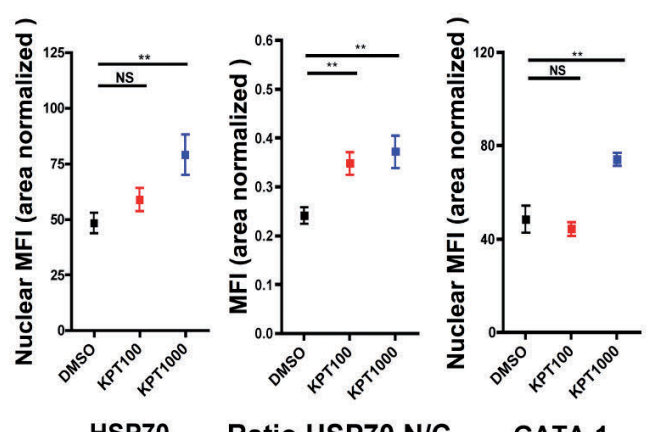

Ratio HSP70 N/C

GATA-1
C
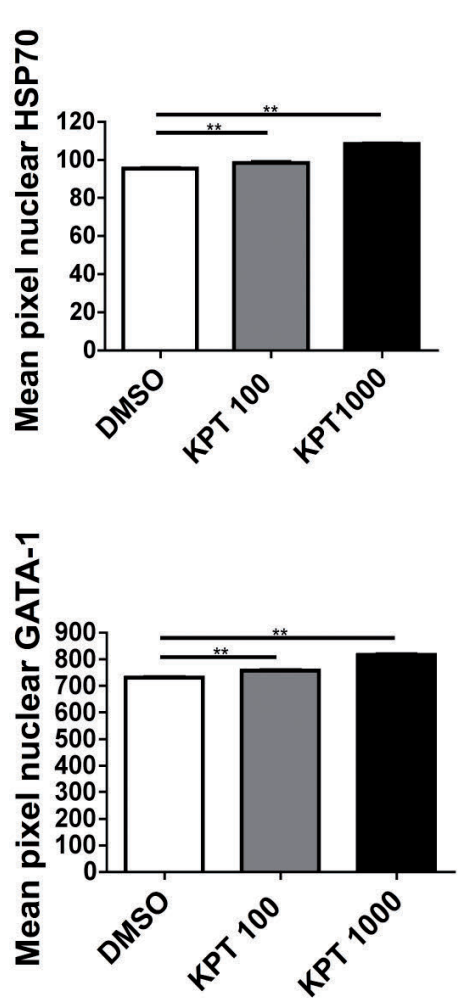

D BTM DMSO

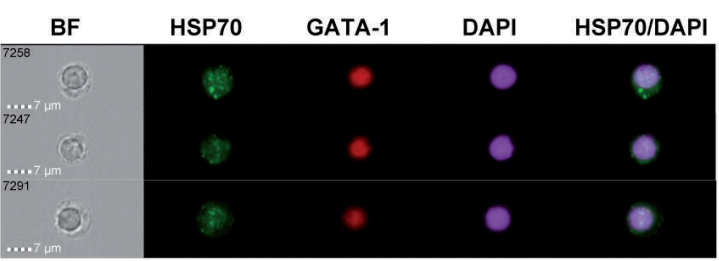

Similarity score $0.748 \pm 0.006$
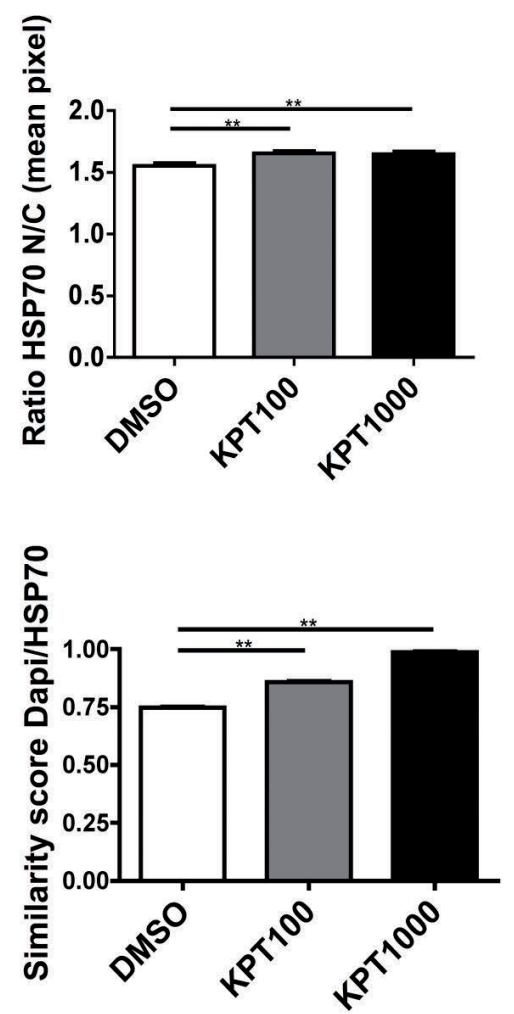

BTM KPT1000

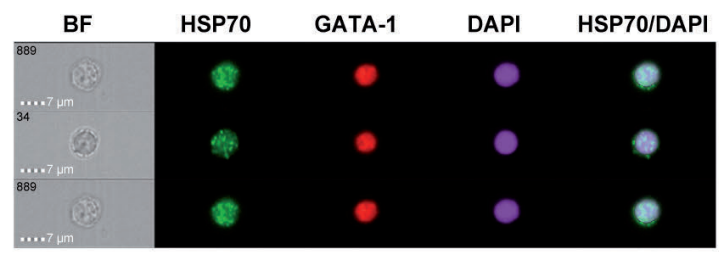

Similarity score $0.987 \pm 0.005$

Figure 3. KPT-251 treatment increases the amount of nuclear HSP70 and GATA-1 in $\beta$-thalassemia major ( $\beta$-TM) erythroid progenitors. Erythroblasts derived from $\beta$-TM peripheral blood cells were treated at day 4 of $\mathrm{CD}^{+} 6^{+}$cell culture with $100 \mathrm{nM}, 1000 \mathrm{nM}$ of KPT-251, or with DMSO (control) for 72 hours (H). All data were analyzed at day 7 of CD36+cell culture (72 hours of treatment). (A) Immunoblot from $10 \mu \mathrm{g}$ of nuclear extracts (NE) and $30 \mu g$ of cytoplasmic extracts (CE), (representative of three independent experiments performed on two different $\beta$-TM patient cell cultures). Graph shows optical relative quantity values of XPO1, HSP70 and GATA1 proteins normalized to that of HSP9O for CE and to that of HDAC2 for NE. Conditions KPT 100nM and 1000nM are normalized to that of DMSO condition. Absence of cytoplasmic proteins contamination in nuclear extracts is evidenced by the absence of HSP9O in NE. (B) Graph shows nuclear mean fluoresence intensity (MFI) of HSP70 and GATA-1, and HSP70 nuclear/cytoplasmic (N/C) ratio of MFI in treated (KPT 100 and 1000) and control (DMSO) cells determined by confocal microscopy images analyses. Data are presented as mean \pm standard error of mean (SEM) (for a minimum of 30 cells per condition), and are normalized on area. $P$ values are determined by ANOVA Dunnett's multiple comparison test. Data are representative of three independent experiments. (C) HSP70 and GATA-1 nuclear expression (mean pixel), HSP70 N/C ratio (mean pixel) and HSP70 nuclear translocation (similarity score) were analyzed by ImageStream. Data are presented in histograms as mean \pm SEM. P-values are determined by ANOVA Dunnett's multiple comparison test (representative of three independent experiments). On average, 30,000 events were collected in all experiments. (D) Three illustrating images of ImageStream experiments. Cells were probed for HSP70 and GATA-1 expression and run on the ImageStream. Bright field (white), HSP70 (green), GATA-1 (red), DAPI (purple) and HSP70/DAPI composite (scale bar=7 $\mu$ m). Respective similarity score \pm SEM are indicated under each group of images. $* P<0.05 ; * * P<0.01$. 
A

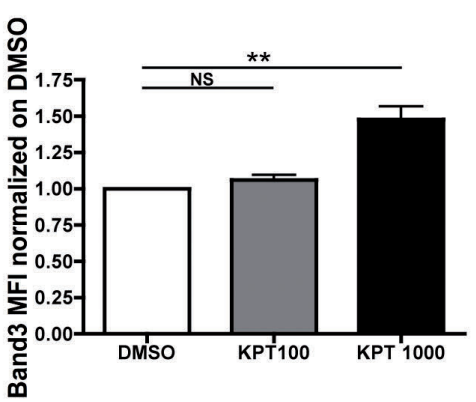

B

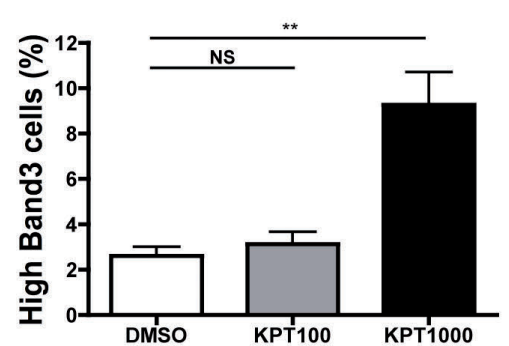

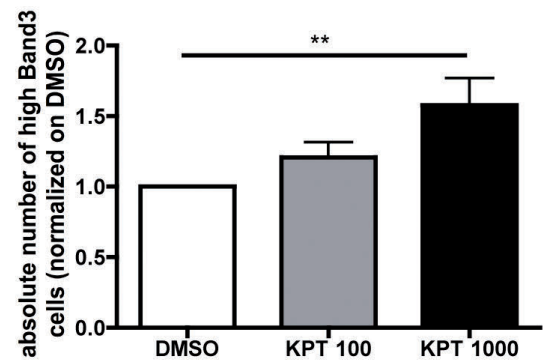

C

BTM H72 DMSO

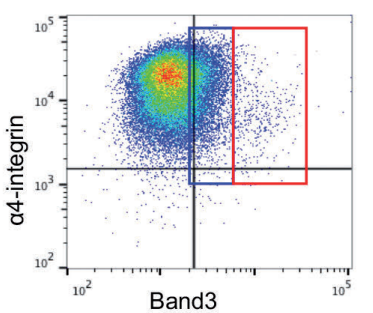

Low Band3 22.8\%

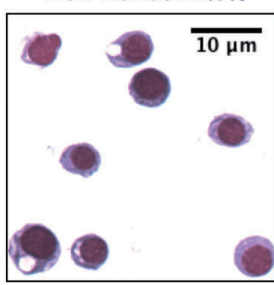

BTM H72 KPT1000

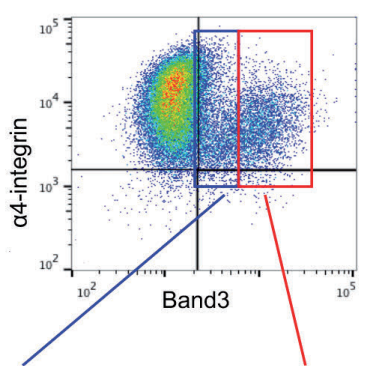

High Band3 62.2\%

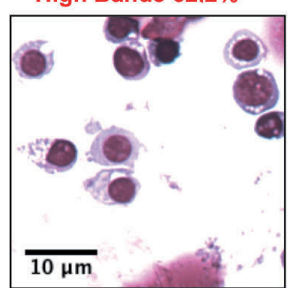

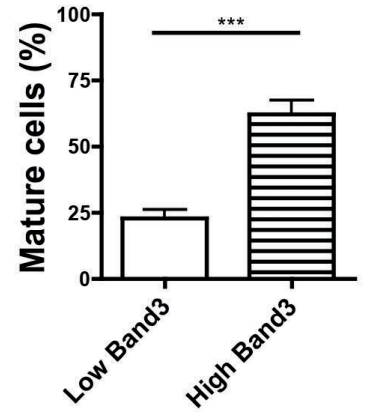

D

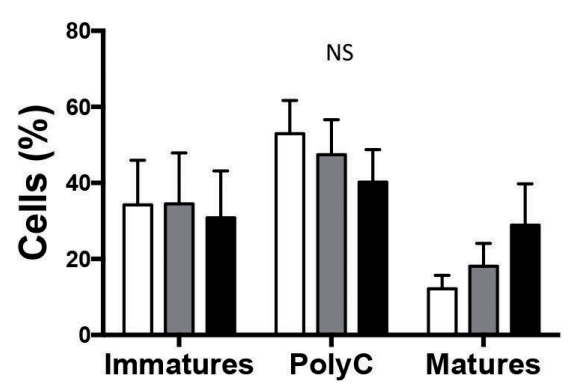

E

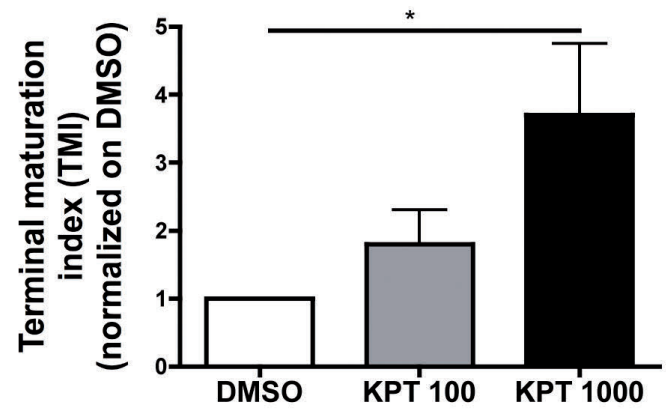

Figure 4. KPT-251 treatment improves terminal erythroid maturation in $\beta$-thalassemia major ( $\beta$-TM) erythroid progenitors in vitro. $\beta$-TM erythroid progenitors were treated at day 4 of $\mathrm{CD}^{2} 6^{+}$cell culture with $100 \mathrm{nM}, 1000 \mathrm{nM}$ of KPT-251, or with DMSO (control) for 72 hours (day 7 CD36 ${ }^{+}$cell culture). (A) The mean fluorescence intensity (MFI) of Band 3 was analyzed by flow cytometry after 72 hours of treatment. MFI were normalized on DMSO condition. $P$-values are determined by ANOVA Dunnett's multiple comparison test $* * P<0.01$, NS: not significant ( $n=8$ independent experiments, $n=3$ different $\beta$-TM samples). (B) (Left) Percentage of high Band3 cell population under the different treatment conditions and (Right) absolute number of high Band3 cells, normalized to DMSO treatment. $P$-values are determined by ANOVA Dunn's multiple comparison test, $* * P<0.01$, NS: not significant, $\mathrm{n}=8$ independent experiments, $\mathrm{n}=3 \beta$-TM patients. (C) Representative flow cytometry plots ( $\alpha 4$-integrin and Band3 staining) of $\beta$-TM erythroid progenitors treated with KPT1000nM or DMSO. Strategy for cell sorting purification of high Band3 (red box) and low Band3 (blue box) erythroblasts populations after 72 hours (H) of KPT1000nM or DMSO treatment (day $7 \mathrm{CD}^{+} 6^{+}$cell culture). A representative morphological analysis (x25 oil objective, scale bar $=10 \mu \mathrm{m}$ ) of purified cells from each gate by May-Grünwald-Giemsa staining. Corresponding graph showing the percentage of mature cells (orthochromatic erythroblasts + reticulocytes) contained in low Band 3 and in high Band 3 gates ( $\mathrm{n}=3$ independent experiments, $\mathrm{n}=2$ different $\beta$-TM patients). $P$-values are determined by paired $t$-test $* * * P<0.001$. (D) Proportions (\%) of immature, polychromatophilic (PolyC), and mature (orthochromatic erythroblasts + reticulocytes) cells after 72 hours of treatment with KPT100nM, KPT 1000nM or DMSO. NS: not significant. (E) Corresponding TM index for the different conditions of treatment. $P$ values are determined by ANOVA Dunnett's multiple comparison test $* P<0.05$, NS: not significant ( $n=5$ independent experiments). 


\section{Differentiation progression}

\section{XP01 expression}

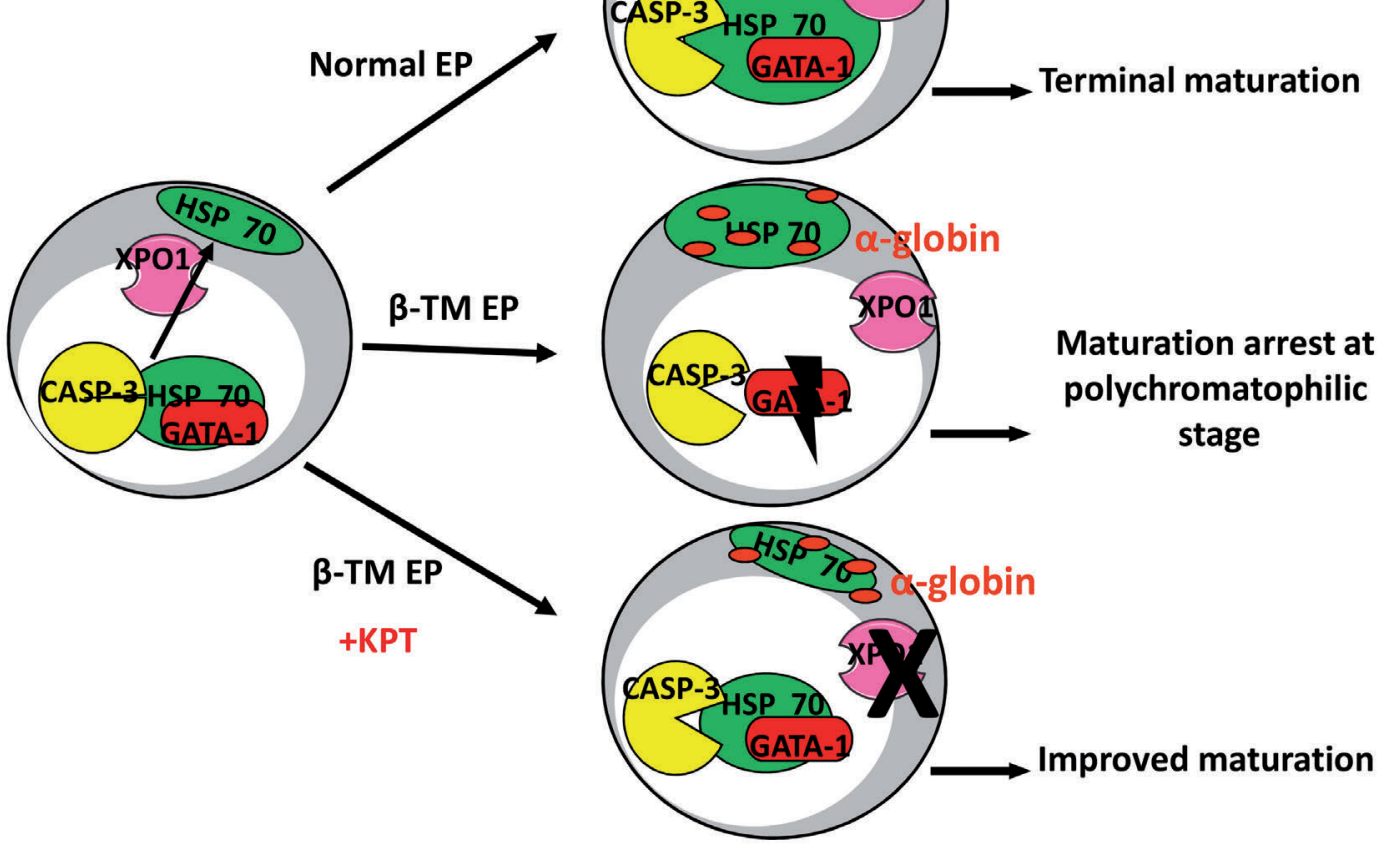

Figure 5. Schematic illustration of the molecular mechanisms modulated by KPT treatment in $\beta$-thalassemia major ( $\beta$-TM) erythroid progenitors compared to $\beta$-TM and normal erythroid progenitors in normal conditions. Schematic representation of molecular mechanisms in normal erythroid progenitor (EP), $\beta$-TM EP, and $\beta$-TM EP treated with KPT (cells on the right). The big arrow on the top represents the direction of differentiation progression. The decrease in XPO1 protein expression is represented by the pink triangle. During early differentiation stages (cell on the left), XPO1 exports HSP70 from the nucleus to the cytoplasm, while XPO1 expression is high. The entry of HSP70 being constant and mediated by Hikeshi, HSP7O is localized both in the cytoplasm and the nucleus at this stage. In normal EP, along differentiation, while XPO1 expression decreases, HSP70 accumulates in the nucleus until caspase-3 activates, corresponding to basophilic stage. Nuclear HSP70 protects GATA1 from caspase- 3 cleavage to enable terminal maturation. In $\beta$-TM EP, at the stage of caspase-3 activation, HSP70 is trapped in the cytoplasm by the excess of free $\alpha$-globin chains and can not protect GATA1 from cleavage. This results in maturation arrest at the polychromatophilic stage. In $\beta$-TM EP treated with KPT, XPO1 activity is repressed. This allows nuclear retention of the small amount of HSP70 that managed to get into the nucleus despite cytoplasm trapping by $\alpha$ chains. At the moment of caspase-3 activation, HSP70 is present in sufficient amount to protect GATA1 and enable an improvement in $\beta$-TM EP terminal maturation.

lowing KPT-251 treatment [similarity score $0.7483 \pm 0.006$ (control), $0.8587 \pm 0.005 \quad(\mathrm{KPT} 100 \mathrm{nM}) \quad(P<0.01)$ and $0.9872 \pm 0.005$ (KPT1000nM) $(P<0.01)]$.

To further demonstrate that XPO1 is indeed the main protein involved in HSP70 nuclear export, we tested its role in HeLa cells, which upon heat shock at $43^{\circ} \mathrm{C}$ exhibited nuclear HSP70 localization as a consequence of both an increase in HSP70 nuclear inflow rate due to an increase of Hikeshi expression and a reduction in nuclear outflow by an unknown mechanism. ${ }^{24}$ After a 6-h recovery phase at $37^{\circ} \mathrm{C}$, the outflow rate increases and HSP70 progressively re-localizes in the cytoplasm. ${ }^{24}$ To demonstrate the role of XPO1 in the nuclear export of HSP70, HSP70 outflow following heat shock was analyzed by ImageStream with or without XPO1 repression (24 h KPT251 pre-treatment at $1000 \mathrm{nM})$. As expected, heat shock induced increased nuclear HSP70 localization and after $6 \mathrm{~h}$ of recovery at $37^{\circ} \mathrm{C}$, HSP70 exited the nucleus, which was delayed when cells have been pre-treated with KPT-251 as compared to control treated cells (Online Supplementary
Figure S3). This observation suggests that XPO1 is the main exportin of HSP70.

Chemical inhibition of XP01 using KPT-251 treatment ameliorates erythroid terminal differentiation of $\beta$-TM erythroid progenitors with low cytotoxicity

As expected, this nuclear accumulation of both HSP70 and GATA-1 was associated with an increase in terminal erythroid differentiation of $\beta$-TM erythroblasts as assessed by flow cytometry analysis showing a significant increase in total Band3 $\mathrm{MFI}^{9}$ [MFI normalized on DMSO treated cells used as a control: $\mathrm{DMSO}=1, \mathrm{KPT} 100 \mathrm{nM}=1.06 \pm 0.04$ (NS), and KPT1000nM=1.48 $\pm 0.09(P<0.01)$ ] (Figure 4A). Consistent with an increase in terminal erythroid maturation, the fraction of cells expressing high Band3 was also increased by KPT treatment in a dose-dependent manner [2.62\% $\% 0.39$ (control), 3.13\% \pm 0.53 (KPT100nM) (NS) and $9.27 \% \pm 1.44$ (KPT 1000nM) $(P<0.01)$ ] (Figure 4B, left). The absolute number of high Band3 cells was also increased (Figure $4 \mathrm{~B}$, right). To ensure that this high Band3 pool rep- 
resented orthochromatic erythroblasts and reticulocytes, as previously described, ${ }^{3}$ we sorted them by FACS based on their Band3 and 4-integrin expression (Figure 4C). As expected, mature erythroblast content (orthochromatic erythroblasts+reticulocytes) was enriched in high Band3 gate $(62.23 \% \pm 5.31)$ compared to low Band3 gate $(22.83 \% \pm 3.49)(P=0.0004)$.

We further confirmed that in $\beta$-TM progenitors, KPT treatment alleviated the maturation arrest at the polychromatophilic stage as it decreased the proportion of polychromatophilic cells and increased the proportion of mature cells (Figure 4D). More importantly, KPT treatment significantly increased the terminal maturation index (TMI), determined as the ratio of orthochromatic erythroblasts+reticulocytes $* 100 /$ polychromatophilic cells, at the dose of 1000nM KPT [TMI normalized to control DMSO: $\quad \mathrm{DMSO}=1, \quad \mathrm{KPT} 100 \mathrm{nM}=1.8 \pm 0.5 \quad$ (NS) KPT1000nM=3.7 $\pm 1.05,(P<0.05)]$ (Figure 4E). Finally, we show that the significant increase in TMI and in absolute number of mature cells at $1000 \mathrm{nM}$ is associated with a high increase in $\mathrm{HbF}$ amount in the pool of mature cells, which is not observed at lower doses of treatment. This effect was observed with a greater magnitude at higher dose $(1500 \mathrm{nM})$. However at this dose, inhibition of cell proliferation and apoptosis were higher (Online Supplementary Figure S4).

In Figure 5, we schematically illustrate the molecular mechanisms modulated by KPT treatment in $\beta$-TM erythroid progenitors compared to $\beta$-TM and normal erythroid progenitors in normal conditions.

\section{Discussion}

Taken together, our data demonstrate that XPO1 is the main nuclear exporter of HSP70 in various cell types, and it may participate in the regulation of HSP70 in human erythroblasts during normal and pathological erythropoiesis. Decreased expression of XPO1 along erythroid differentiation provides an explanation for the augmentation of nuclear localization of HSP70 during normal erythroid maturation. Interestingly, proteins of the Ran machinery (RAN, RANBP1, RCC1, NUTF1 AND RAN-
GAP1) essential for XPO1 activity are also expressed during erythroid differentiation and also down-regulated at the time of caspase activation (data not shown). Furthermore, we demonstrate that inhibition of XPO1 by KPT-251 treatment improves terminal maturation of $\beta$ TM erythroblasts by preventing GATA-1 degradation by caspase-3. Thus, XPO1 inhibitors could be added to the armamentarium of therapeutic options of $\beta$-TM to correct ineffective erythropoiesis and its pathological consequences (e.g. iron overload and extramedullary hematopoiesis), to increase hemoglobin level, to reduce transfusion burden and ultimately to increase overall survival. Interestingly, KPT compounds are currently under clinical development and exhibit high efficacy and safety profile in cancer therapy. More recently, a new generation of KPT compounds with reduced toxicity is being developed (clinicaltrials.gov identifier: NCT02649790) and as such, if shown to be not toxic in the long-term, they could be used in $\beta$-TM as well. In addition, maintaining HSP70 in the nucleus by blocking XPO1 could be useful in other pathologies of erythropoiesis where HSP70 is not localized in the nucleus, such as myelodysplastic syndrome ${ }^{25}$ and congenital erythroblastopenia, ${ }^{26}$ but also in other diseases such as some neurodegenerative diseases, in which protein aggregates may also prevent HSP70 nuclear location.

\section{Acknowledgments}

The authors would like to thank the Department of Biotherapy and the Maternity at the Necker Hospital (Paris, France) for providing blood samples. This work was supported by the French National Research Agency ANR-13-BSV1-0029-05 "HSPathies", LabEx GRex financial support ANR-11-IDEX-O, the French National Research Agency (ANR) under the program "Investissements d'Avenir" (LabExGRex and LipSTIC), the Institut National du Cancer (INCa), the Ligue Nationale Contre le Cancer ("Label of Excellence"), Ministère de l'Enseignement Supérieur et de la Recherche, FEDER and Région Bourgogne.

\section{Funding}

This program has received a state subsidy managed by the National Research Agency under the "Investments for the Future" program bearing the reference ANR-01-AO-IAHU.

\section{References}

1. Zermati Y, Garrido C, Amsellem S, et al. Caspase activation is required for terminal erythroid differentiation. J Exp Med. 2001; 193(2):247-254

2. Ribeil J-A, Zermati Y, VandekerckhoveJ, et al. Hsp70 regulates erythropoiesis by preventing caspase-3-mediated cleavage of GATA-1. Nature. 2007;445(7123):102-105.

3. Arlet J-B, Ribeil J-A, Guillem F, et al. HSP70 sequestration by free $\alpha$-globin promotes ineffective erythropoiesis in $\beta$-thalassaemia. Nature. 2014;514(7521):242-246.

4. Kudo N, Wolff B, Sekimoto $T$, et al. Leptomycin B inhibition of signal-mediated nuclear export by direct binding to CRM1. Exp Cell Res. 1998;242(2):540-547.

5. Nishi K, Yoshida M, Fujiwara D, Nishikawa M, Horinouchi S, Beppu T. Leptomycin B targets a regulatory cascade of crm1, a fission yeast nuclear protein, involved in control of higher order chromosome structure and gene expression. J Biol Chem. 1994; 269(9):6320-6324.

6. Lui K, Huang Y. RanGTPase: a key regulator of nucleocytoplasmic trafficking. Mol Cell Pharmacol. 2009;1(3):148-156.

7. Hattangadi SM, Martinez-Morilla S, Patterson HC, et al. Histones to the cytosol: exportin 7 is essential for normal terminal erythroid nuclear maturation. Blood. 2014; 124(12):1931-1940.

8. Mathias LA, Fisher TC, Zeng L, et al. Ineffective erythropoiesis in beta-thalassemia major is due to apoptosis at the polychromatophilic normoblast stage. Exp Hematol. 2000;28(12):1343-1353.

9. Hu J, Liu J, Xue F,et al. Isolation and functional characterization of human erythroblasts at distinct stages: implications for understanding of normal and disordered erythropoiesis in vivo. Blood. 2013; 121(16): 3246-3253.

10. An X, Schulz VP, Li J, et al. Global transcriptome analyses of human and murine terminal erythroid differentiation. Blood. 2014;1 23(22):3466-3477.

11. Gautier E-F, Ducamp S, Leduc M, et al. Comprehensive Proteomic Analysis of Human Erythropoiesis. Cell Rep. 2016; 16(5):1470-1484

12. Fukuda M, Asano S, Nakamura T, et al. CRM1 is responsible for intracellular transport mediated by the nuclear export signal. Nature. 1997;390(6657):308-311.

13. Xu D, Farmer A, Chook YM. Recognition of nuclear targeting signals by Karyopherin- $\beta$ proteins. Curr Opin Struct Biol. 2010; 20(6):782-790

14. Pinello L, Xu J, Orkin SH, Yuan G-C. Analysis of chromatin-state plasticity iden- 
tifies cell-type-specific regulators of H3K27me3 patterns. Proc Natl Acad Sci U S A. 2014;111(3):344-353.

15. Etchin J, Sun $Q$, Kentsis A, et al. Antileukemic activity of nuclear export inhibitors that spare normal hematopoietic cells. Leukemia. 2013;27(1):66-74

16. Lapalombella R, Sun $Q$, Williams $K$, et al. Selective inhibitors of nuclear export show that CRM1/XPO1 is a target in chronic lymphocytic leukemia. Blood. 2012; 120(23): 4621-4634.

17. Etchin J, Sanda T, Mansour MR, et al. KPT330 inhibitor of CRM1 (XPO1)-mediated nuclear export has selective anti-leukaemic activity in preclinical models of T-cell acute lymphoblastic leukaemia and acute myeloid leukaemia. $\mathrm{Br} J$ Haematol. 2013;161(1):117-127.

18. Kojima K, Kornblau SM, Ruvolo V, et al. Prognostic impact and targeting of CRM1 in acute myeloid leukemia. Blood. 2013; 121(20):4166-4174

19. Walker CJ, Oaks JJ, Santhanam R, et al. Preclinical and clinical efficacy of XPO1/CRM1 inhibition by the karyopherin inhibitor KPT-330 in Ph+ leukemias. Blood. 2013;122(17):3034-3044.

20. Wettersten HI, Landesman Y, Friedlander S, Shacham S, Kauffman M, Weiss RH Specific inhibition of the nuclear exporter exportin-1 attenuates kidney cancer growth. PloS One. 2014;9(12):1-15.

21. Ranganathan P, Yu X, Na C, et al. Preclinical activity of a novel CRM1 inhibitor in acute myeloid leukemia. Blood. 2012;120(9):1765-1773

22. Gravina GL, Mancini A, Sanita P, et al. KPT-330, a potent and selective exportin-1 (XPO-1) inhibitor, shows antitumor effects modulating the expression of cyclin D1 and survivin [corrected] in prostate cancer models. BMC Cancer. 2015;15:941-960.

23. Tai Y-T, Landesman Y, Acharya C, et al. CRM1 inhibition induces tumor cell cytotoxicity and impairs osteoclastogenesis in multiple myeloma: molecular mechanisms and therapeutic implications. Leukemia. 2014;28(1):155-165

24. Zeng X-C, Bhasin S, Wu X, et al. Hsp70 dynamics in vivo: effect of heat shock and protein aggregation. J Cell Sci. 2004;117(Pt 21):4991-5000.

25. Frisan E, Vandekerckhove I, de Thonel A et al. Defective nuclear localization of Hsp70 is associated with dyserythropoiesis and GATA-1 cleavage in myelodysplastic syndromes. Blood. 2012;119(6): 1532-1542.

26. Gastou M, Rio S, Dussiot M, et al. The severe phenotype of Diamond-Blackfan anemia is modulated by heat shock protein 70. Blood Adv. 2017;1(22):1959-1976. 